\title{
Overcoming PARP Inhibitor and Platinum Resistance with WEE1 Inhibitors in Ovarian Cancer
}

\author{
Ilaria Colombo ${ }^{1 *}$
}

\section{ABSTRACT}

Poly (ADP-ribose) polymerase inhibitors (PARPi) have transformed the treatment of high-grade epithelial ovarian cancer (EOC), with demonstrated efficacy in the recurrent as well as newly diagnosed disease. However, women with EOC inevitably acquire resistance to both platinum chemotherapy and PARPi. Combining PARPi with agents targeting other DNA damage response (DDR) pathways to promote synthetic lethality is a rational therapeutic approach. Recent studies show that combination of PARPi and WEE1 inhibitors is feasible and might be an effective strategy for patients with platinum-and PARPi-resistant EOC. This article provides an overview of the latest preclinical and clinical trials evaluating WEE1 inhibitors in monotherapy or in combination with PARPi in advanced EOC.

Keywords: ovarian cancer, PARP, platinum, WEE1, resistance, DNA damage response

\section{INTRODUCTION}

Ovarian cancer $(\mathrm{OC})$ is the $7^{\text {th }}$ most common type of cancer in women worldwide and the $5^{\text {th }}$ most common cause of cancer-related death in women in Western nations. ${ }^{1}$ Overall prognosis is poor, with a 5 -year relative survival rate of $30.2 \%$ for advanced-stage disease. ${ }^{2}$ Delay in diagnosis is a clinically significant issue, with at least $70 \%$ of OC patients having International Federation of Gynecology and Obstetrics (FIGO) stage III or IV disease at diagnosis. ${ }^{3}$

Epithelial ovarian cancer (EOC) represents approximately $90 \%$ of all $\mathrm{OC}^{1}$ and comprised four histological subtypes (serous, mucinous, clear-cell, and endometrioid) based upon their distinct tissue architecture and morphology. High-grade serous ovarian cancer (HGSOC) is the most common EOC histotype. ${ }^{4}$ Different OC subtypes have different molecular characteristics, which in turn influence current standard treatment. ${ }^{5}$

\section{TREATMENT OF ADVANCED EOC WITH PARP INHIBITORS}

Optimal cytoreductive surgery to no residual disease followed by platinum-based chemotherapy remains the gold-standard treatment for newly diagnosed EOC. ${ }^{6,7}$ For patients with highrisk disease (residual tumor after primary debulking surgery or stage IV), the addition of the anti-angiogenic agent bevacizumab has improved patients' outcome and became part of the standard of care. ${ }^{6}$ Ultimately, even with an excellent initial response, more than $80 \%$ of patients with advanced-stage EOC will experience disease recurrence.

Poly (ADP ribose) polymerase inhibitors (PARPi) are newer drugs that have transformed the treatment paradigm of EOC. ${ }^{8}$ PARPi have shown to improve patients outcome in different settings: maintenance treatment in first-line ${ }^{9-11}$, maintenance in platinum-sensitive recurren$\mathrm{ce}^{12-14}$ or as a new line of treatment ${ }^{15,16}$. Thus, most patients with EOC will receive a PARPi treatment at some point during their disease course. Despite these promising results, many patients will inevitably develop primary or acquired resistance to PARPi and as of today, no approved agents are available to effectively treat patients after progression to these targeted agents. Different mechanisms of PARPi resistance have been proposed, some of them similar to the ones responsible for platinum resistance. ${ }^{17,18}$ Understanding these mechanisms and developing new therapies to overcome them is a key priority for current clinical and translational research in ovarian cancer.

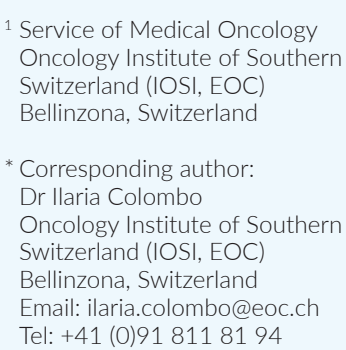

DOI: 10.36000/hbT.OH.2021.09.047 ISSN: 2673-2092 (Print) and 2673 2106 (Online)

This article was received on August 24, 2021.

This article was accepted on September 15, 2021.

This article was published on October 15, 2021.

Colombo I. Overcoming PARP Inhibitor and Platinum Resistance with WEE1 Inhibitors in Ovarian Cancer healthbook TIMES Onco Hema. 2021;(9):38-43. 


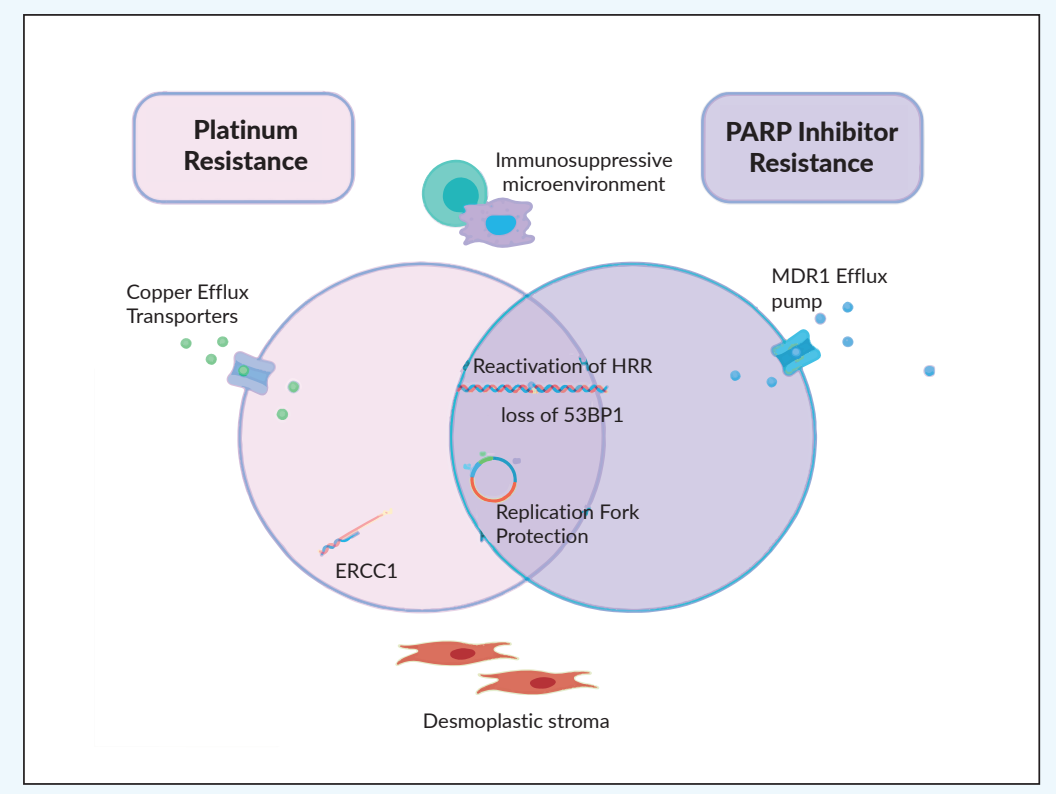

Figure 1. Overlapping mechanisms of resistance to platinum-based and PARP inhibitor treatment. While both mechanisms demonstrate reactivation of HRR repair and loss of 53BP1 and replication fork protection promoting progression through the cell cycle, platinum resistance is additionally associated with defects in the NER pathway, including increased ERCC1 activity. ERCC1. excision repair cross-complementing group 1 protein; $H R R$, homologous recombination repair; MDR1, multidrug resistance protein 1; NER, nucleotide excision repair; PARP, poly (ADP-ribose) polymerase; 53BP1, p53 binding protein 1. Adapted from McMullen et al. 2020.17

\section{MECHANISMS OF PARPI RESISTANCE}

Homologous recombination (HR) has been identified as one of the main pathways involved in repairing double-strand (DSB) DNA breaks resulting from DNA damage. ${ }^{19}$ In tumor cells with defective HR (homologous recombination deficiency, HRD) repair pathways, PARP modulates repair of DNA single-strand (SBB) breaks and thus enables cell survival. ${ }^{17-19}$ In tumor cells with HRD, PARPi trap PARP on DNA to block SSB repair, promoting the accumulation of fatal DSB and DNA damage and triggering cell death through the welldescribed mechanism of synthetic lethality. ${ }^{19-21}$

Approximately $50 \%$ of all EOCs are characterized by HRD, mainly due to genomic alterations in HR genes, including but not limited to $B R C A 1 / 2$, and this correlates with a higher sensitivity to platinum-based chemotherapy and PARPi. ${ }^{22}$ Nevertheless, PARPi are less effective in cancer patients with proficient HR tumors. ${ }^{10,11,13}$ Thus, HRD and BRCA status are now established biomarkers for predicting responsiveness to both platinum chemotherapy and PARPi. Different HRD tests have been developed (e.g., tumor mutations in HR genes, genomic scars assessment [myChoice Myriad HRD test, FoundatioFocus CDxBRCA, Foundation Medicin] or functional assays) to predict the magnitude of benefit from PARPi. However, these currently available tests have a low negative predictive value and more reliable assays are urgently needed. ${ }^{23}$

Different mechanisms sustaining PARPi resistance have been described. One of the major mechanisms of PARPi and platinum-based chemotherapy resistance is the restoration of HR capability re-enabling cancer cells to repair DSBs, thereby reducing genomic instability and sensitivity to PARPi (Figure 1). ${ }^{17,18}$ Restoration of HR might occur due to epigenetic events leading to re-expression of HR genes or to secondary mutations that re-established gene function (reversion mutations) or upregulation of the wild-type allele. ${ }^{24-26}$ For example, somatic $B R C A$ reversion mutations that restore BRCA function have been reported and is now a well-established mechanism of PARPi resistance. ${ }^{24-26}$ Other mechanisms include reactivation of HR due to loss of DDR p53binding protein [53BP1], involved in the error-prone non-homologous end-joining repair ${ }^{27}$, and stabilization of replication fork.

Another possible mechanisms of resistance are the activation of other pathways (PI3K/AKT/mTOR, RAS/RAF/MET, VEGFR) able to activate or restore $\mathrm{HR}$ and the increased dependence on other DNA repair mechanisms or cell cycle regulators. ${ }^{28,29}$ The cell cycle checkpoints (e.g. ataxia telangiectasia mutated protein $[\mathrm{ATM}]$, the ataxia telangiectasia and Rad3related protein [ATR], p53, WEE1, checkpoint kinase 1 and 2 [CHK1 and CHK2]) coordinate the arrest of the cell cycle to repair the DNA damages and promote a high-fidelity cell replication. ${ }^{30}$

Notably, about $95 \%$ of HGSOC harbor mutant TP53, a tumor suppressor gene involved in regulating the first $\mathrm{G} 1 / \mathrm{S}$ cell cycle checkpoint. Tumor cells deficient in the first checkpoint are more dependent on the G2/M checkpoint and the WEE1 pathway for DNA repair.

\section{WEE1 KINASES AND INHIBITORS}

\section{Mechanisms of action}

WEE1 is an inhibitory kinase that plays an important role in cell cycle regulation and DDR, particularly as a gatekeeper of the G2/M stage of the cell cycle (Figure 2).$^{31}$ WEE1 also regulates DNA replication and chromatin integrity during the $S$ phase. In response to DNA damage, WEE1 directly inhibits the downstream cyclin-dependent kinase (CDK) 1 and CDK2 protein complexes, thus preventing mitotic entry and replication of cells with altered DNA. 


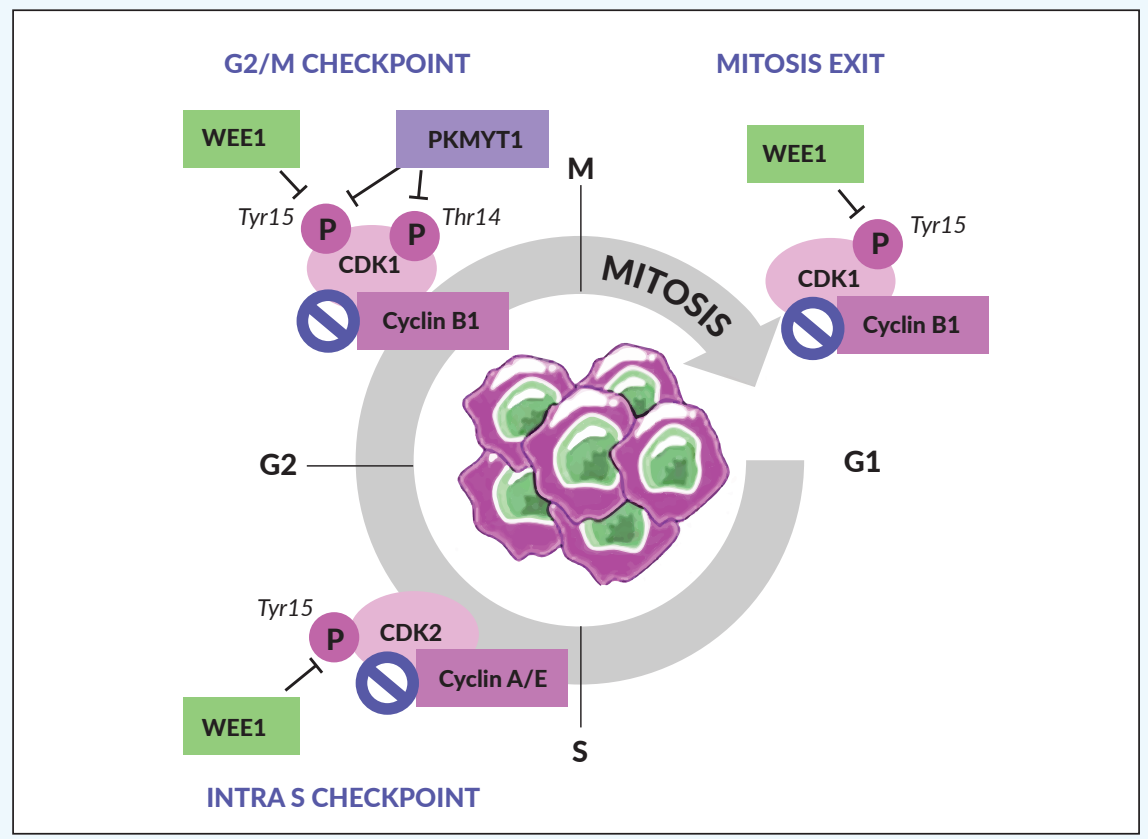

Figure 2. WEE1 mechanism of action. Schematic representation of WEE1 involvement in cell cycle checkpoints. CDK, cyclindependent kinase; G2/M, gap 2/mitosis phase; $\mathrm{S}$, synthesis phase; PKMYT1, membrane-associated tyrosine- and threonine-specific cdc2inhibitory kinase; WEE1, WEE1 tyrosine kinase. Adapted from Ghelli Luserna di Rorà et al. $2020 .{ }^{31}$

WEE1, therefore, acts as a tumor suppressor in healthy, nonmalignant cells. ${ }^{31}$ Aberrant WEE1 gene expression has been observed in various human cancers. For example, WEE1 gene overexpression has been found in 35\% of breast cancers, especially in human epidermal growth factor receptor 2 (HER2)positive subtypes, and $92 \%$ of OCs. ${ }^{32,33}$ In contrast, WEE1 gene suppression was found in human colon cancer cell lines analyzed using complementary DNA (cDNA) array techniques. ${ }^{34}$ WEE1 inhibition removes the brakes on cell cycle progression and increases DNA damage, which leads to catastrophic mitosis and tumor cell death. Together, these observations provided the rationale for investigating WEE1 inhibition as a viable therapeutic anticancer strategy. ${ }^{35-37}$

\section{OVERCOMING PARPI AND PLATINUM RESISTANCE WITH WEE 1 INHIBITORS}

Preclinical and clinical evidence for combining WEE1 inbibitors with chemotherapy

Impacting both DDR and cell cycle progression, WEE1 inhibition may increase cell death in response to chemotherapy and ionizing radiation. A proof-of-concept study in pancreatic cancer cells showed that WEE1 inhibition sensitized HR-proficient (BRCA2 wild-type) cancers but not HRD-positive (BRCA2 mutant) cancers to gemcitabine-based chemoradiation. ${ }^{38}$ This preclinical study suggests that WEE1 inhibitors are involved in the inhibition of HR repair as well as abrogation of the $\mathrm{G} 2$ checkpoint. ${ }^{38}$

Adavosertib, a first-in-class selective small-molecule inhibitor of WEE, prevents WEE1 from phosphorylating the Tyr 15 residue of CDK1, thus activating the cyclin B1-CDK1 complex to enable cells to enter mitosis. ${ }^{31}$ Evasion of the G2 phase forces cells harboring DNA lesions to progress into mitosis, inducing premature mitotic exit and apoptosis. ${ }^{31,37}$ Early-phase clinical trials in several adult solid tumors have shown that adavo- sertib has promising antitumor activity as monotherapy or in combination with chemotherapy drugs such as 5-fluorouracil (5-FU), gemcitabine, carboplatin and cisplatin. ${ }^{39-41}$

Several clinical studies have investigated the strategy of synthetic lethality by exploring simultaneous inhibition of multiple DNA repair mechanisms, for example, dual inhibition of WEE1 and PARP in combination with other DNA-damaging agents in patients with tumors harboring $B R C A$ mutations or the combination of a WEE1 inhibitor and chemotherapy. ${ }^{42-45}$ Notably, clinical evidence for sensitization of platinum-resistant EOC cells to platinum-based chemotherapy has been demonstrated with WEE1 inhibitors (Table 1). A phase II study reported by Leijen et al. (2016) provided clinical proof that the WEE1 inhibitor, adavosertib, could enhance the efficacy of carboplatin in patients with TP53-mutated EOC refractory or resistant ( $<3$ months) to first-line platinum-based therapy. ${ }^{42}$ Another randomized phase II placebo-controlled study investigated the role of adding adavosertib to carboplatin and paclitaxel in patients with TP53-mutated platinum-sensitive recurrent EOC, showing an improvement in progression-free survival (PFS) (9.9 months vs 8.0 months, HR: 0.55 [95\% CI: $0.32-$ $0.95]) .{ }^{45}$ Furthermore, in a double-blind, randomized, placebocontrolled phase II study in patients with platinum-resistant or platinum-refractory advanced HGSOC, adavosertib combined with gemcitabine significantly improved PFS (4.6 months vs 3.0 months, HR 0.55 [95\% CI: 0.35-0.90]) and overall survival (OS) (11.4 months vs 7.2 months, HR: 0.56, [95\% CI: $0.35-$ $0.91]$ ) versus placebo. ${ }^{43}$ Notably, signs of activity of adavosertib plus gemcitabine were also observed in an exploratory cohort of rare histological subtypes, including low-grade serous or endometrioid, carcinosarcoma or clear cell OC. ${ }^{43}$ As expected, the combination of adavosertib and chemotherapy resulted in increased toxicity, mainly hematological that frequently required dose interruption or reduction. . $^{42,43,45}$ 


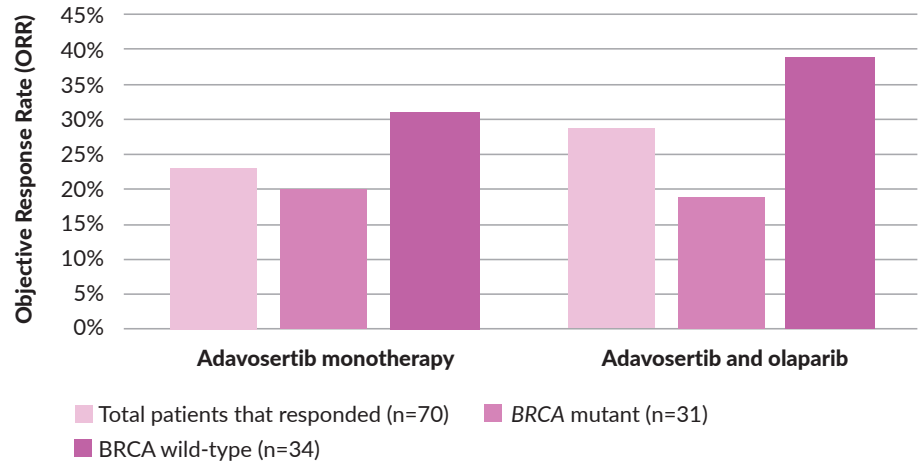

Figure 3. Response to adavosertib therapy in PARP-resistant ovarian cancer in the EFFORT trial. Adapted from Westin et al. 2021.44

Preclinical and clinical evidence for combining WEE1 and PARP inhibitors to overcome PARPi resistance

Preclinical studies showed that the inhibition of WEE1 might reverse resistance to PARPi. In pancreatic cancer cells, the addition of a WEE1 inhibitor in HR-proficient tumors enabled maximal radiosensitization by PARPi. ${ }^{46}$ Therefore, these two agents may work in synergy to impair DNA replication at multiple levels.

The benefit of dual inhibition of HR repair pathways in patients with advanced OC using a combination of WEE1 inhibitors and PARPi was recently examined in the EFFORT (EFFicacy off adavosertib in parp ResisTance) study (Table 1). EFFORT (clinical trial identifier: NCT03579316) is a randomized, two-arm, non-comparative phase II study that evaluated adavosertib with or without the PARPi olaparib in 80 women with PARPi-resistant OC. ${ }^{44}$ The overall response rate (ORR) was $23 \%$ for patients in the adavosertib monotherapy arm and
$29 \%$ in the combination arm (Figure 3). The clinical benefit rate (objective response and stable disease $>4$ months) for patients receiving adavosertib alone or in combination with olaparib was $63 \%$ and $89 \%$, respectively. Median PFS was 5.5 months (90\% CI: 3.9-6.9) in the monotherapy arm and 6.9 months (90\% CI: 4.3-8.3) in the combination arm. ${ }^{44}$ Efficacy of adavosertib as a single agent or in combination with olaparib was demonstrated regardless BRCA status. Among women with $B R C A$ mutations, the ORR was $20 \%$ with adavosertib monotherapy $(\mathrm{n}=15)$ versus $19 \%$ with the combination $(n=16)$ (Figure 3), with a median duration of response of 5.6 months and 6.4 months, respectively, and clinical benefit rate of $67 \%$ and $81 \%$, respectively. In patients with BRCA wildtype, ORR was $31 \%$ with adavosertib $(n=16)$ and $39 \%$ with adavosertib and olaparib $(n=18)$ and the clinical benefit rate of $69 \%$ versus $94 \%$. The median duration of response was 4.1 and 8.7 months, respectively. ${ }^{44}$

Table1. Summary of the main trials of adavosertib in gynecological malignancies. HR, hazard ratio; N, number; NC, non-calculable; OC, ovarian cancer; ORR, overall response rate; OS, median overall survival; PFS, median progression-free survival.

\begin{tabular}{|c|c|c|c|c|c|}
\hline Treatment & Phase & Cancer type & $N$ & Efficacy & Ref \\
\hline $\begin{array}{l}\text { Adavosertib + } \\
\text { carboplatin }\end{array}$ & $\begin{array}{l}\text { Phase II, } \\
\text { single arm }\end{array}$ & $\begin{array}{l}\text { TP53-mutant OC } \\
\text { refractory or resistant }(<3 \\
\text { months) to first-line platinum- } \\
\text { chemotherapy }\end{array}$ & 24 & $\begin{array}{l}\text { ORR: } 43 \% \\
\text { PFS: } 5.3 \text { months } \\
\text { OS: } 12.6 \text { months }\end{array}$ & $\begin{array}{l}\text { Leijen et } \\
\text { al. }{ }^{42}\end{array}$ \\
\hline $\begin{array}{l}\text { Adavosertib } \\
\text { or placebo + } \\
\text { carboplatin/ } \\
\text { paclitaxel }\end{array}$ & $\begin{array}{l}\text { Phase } 11 \text {, } \\
\text { randomized }\end{array}$ & $\begin{array}{l}\text { TP53-mutant OC } \\
\text { platinum-sensitive }\end{array}$ & 121 & $\begin{array}{l}\text { ORR: } 74.6 \text { vs } 69.9 \%(p=0.52) \\
\text { PFS: } 9.9 \text { vs } 8 \text { months (HR: } 0.55) \\
\text { OS: NC vs } 35.4 \text { months (HR: } 1.0)\end{array}$ & $\begin{array}{l}\text { Oza et } \\
\text { al. }^{45}\end{array}$ \\
\hline $\begin{array}{l}\text { Adavosertib } \\
\text { or placebo + } \\
\text { gemcitabine }\end{array}$ & $\begin{array}{l}\text { Phase } 11 \text {, } \\
\text { randomized }\end{array}$ & $\begin{array}{l}\text { Platinum-resistant/refractory } \\
\text { HGSOC }\end{array}$ & 99 & $\begin{array}{l}\text { ORR: } 23 \text { vs } 6 \%(p=0.038) \\
\text { PFS: } 4.6 \text { vs } 3 \text { months (HR: } 0.55) \\
\text { OS: } 11.4 \text { vs } 7.2 \text { months (HR: } 0.56)\end{array}$ & $\begin{array}{l}\text { Lheureux } \\
\text { et al. }{ }^{43}\end{array}$ \\
\hline $\begin{array}{l}\text { Adavosertib or } \\
\text { adavosertib + } \\
\text { olaparib }\end{array}$ & $\begin{array}{l}\text { Phase II, } \\
\text { randomized non } \\
\text { comparative }\end{array}$ & PARPi resistant OC & $\begin{array}{l}35 \text { in } \\
\text { each arm }\end{array}$ & $\begin{array}{l}\text { Adavosertib } \\
\text { ORR: } 23 \%, \text { PFS: } 5.3 \text { months } \\
\text { Adavosertib + Olaparib } \\
\text { ORR: } 29 \%, \text { PFS: } 6.8\end{array}$ & $\begin{array}{l}\text { Westin et } \\
\text { al. }\end{array}$ \\
\hline Adavosertib & $\begin{array}{l}\text { Phase II, } \\
\text { single arm }\end{array}$ & $\begin{array}{l}\text { Recurrent serous endometrial } \\
\text { cancer }\end{array}$ & 34 & $\begin{array}{l}\text { ORR: } 29.4 \% \\
\text { PFS: } 6.1 \text { months }\end{array}$ & Liu et al. ${ }^{50}$ \\
\hline
\end{tabular}


Patients in both monotherapy and combination treatment arms experienced treatment-associated adverse events, mainly hematological and gastro-intestinal. Despite these toxicities were usually manageable with supportive care, dose interruptions ( $72 \%$ monotherapy vs $88 \%$ combination arm), or dose reductions ( $51 \%$ vs $71 \%$ ) were necessary. ${ }^{44}$ Further research is warranted to define the optimal dose and schedule of adavosertib in combination with PARPi, to improve tolerability and dose intensity and to maximize patient benefit. Fang et al. (2019) showed that adding a WEE1 inhibitor and PARPi in sequence minimizes toxicity in TP53-mutant and BRCA wild-type OC model while maintaining efficacy and avoid the onset of drug resistance. ${ }^{47} \mathrm{~A}$ phase I trial is ongoing (STAR trial, NCT04197713) to asses the sequential combination of adavosertib and olaparib in patient with advanced solid tumors with selected HR genes mutations and failure to previous treatment with a PARPi.

\section{WEE1 inhibitor in other gynecological malignancies}

The efficacy of the WEE1 inhibitor adavosertib has been also investigated for the treatment of advanced recurrent serous endometrial cancer following failure of platinum-based chemotherapy. Serous uterine cancer is a distinct histological subtype of endometrial cancer with, similarly to HGSOC, an high prevalence of TP53 mutations and high replication stress. ${ }^{48} \mathrm{~A}$ single-arm phase II study investigated the efficacy of adavosertib among 34 evaluable patients, with an ORR of $29.4 \%$ with a median duration of response of 9 months and a PFS of 6.1 months. ${ }^{49}$ Despite these results suggest a possible new therapeutic opportunity for patients with endometrial cancer, further research is needed to define predictive biomarkers and confirm these preliminary findings. An international phase II study is ongoing (ADAGIO, NCT04590248) to assess the efficacy of adavosertib in a larger population of women with serous uterine cancer progressed after at least one prior line of platinum-based chemotherapy.

\section{CONFLICT OF INTEREST}

Travel grants: Tesaro

External expert: AZ, GSK, Novartis

Institutional grants for clinical trials

(PI): MSD, Bayer, Oasmia

\section{CONCLUSIONS}

As the primary G2/M cell-cycle checkpoint gatekeeper, the WEE1 pathway can be used to exploit intrinsic replication stress and dysfunctional checkpoint in tumor cells. Therefore, the rational combination of a WEE1 inhibitor to DNA-damaging chemoradiotherapies or PARPi may be a viable anticancer treatment strategy for various tumor types. Promising preclinical and early clinical trial results show that combined WEE1 inhibition enhances the effect of chemoradiotherapy or PAR$\mathrm{Pi}$ in patients with platinum- or PARP-resistant ovarian cancer regardless of BRCA status. One of the main limitations for the clinical development of this compound, both as single agent and in combination, is represented by the high rate of hematological toxicities, often requiring dose reduction or interruption, particularly in heavily pretreated population. However, the timing of combination therapy is important to obtain optimal treatment efficacy and improve tolerability. Studies determining the optimal regimen and schedule to combine DNA damaging agents and WEE1 inhibitors to treat advanced ovarian cancer are ongoing and the results will provide further insight on how better administered these agents. No biomarkers able to predict WEE1 efficacy and to support a better patient's selection are currently available and further results from translational research are eagerly awaited. Moreover, other WEE1 inhibitors (IMP7068, Debio0123) are under development and will give further information into the potential role of these agents for the treatment of EOC and other solid tumor types.

\section{TAKE-HOME MESSAGES}

- It is possible to combine DNA damage response inhibitors, such as cell cycle checkpoint inhibitors, chemoradiation or PARP inhibitors, to enhance antitumor activity.

- More than $95 \%$ of patients with high-grade serous ovarian cancer harbor mutant TP53, suggesting that the G2/M checkpoint is a potential anticancer target.

- Several preclinical and clinical trials have investigated the efficacy of the WEE1 inhibitor adavosertib as a single agent or in combination with other DNA-damaging agents or PARP inhibitors.

- In patients with PARPi resistant EOC, WEE1 has shown early signs of activity when used as a single agent or in combination with olaparib, regardless of BRCA status, warranted further exploration. 
REFERENCES

1. Lisio MA, Fu L, Goyeneche A, Gao ZH, Telleria C. HighGrade Serous Ovarian Cancer: Basic Sciences, Clinical and Therapeutic Standpoints. Int J Mol Sci. 2019;20(4):952. doi:10.3390/ijms20040952

2. Hinchcliff E, Chelariu-Raicu A, Westin SN. Current and future landscape of poly (ADP-ribose) polymerase inhibition resistance. Curr Opin Obstet Gynecol. 2021;33(1):19-25. doi:10.1097/GCO.0000000000000678

3. Lheureux S, Gourley C, Vergote I, Oza AM. Epithelial ovarian cancer. Lancet. 2019;393(10177):1240-1253. doi:10.1016/ S0140-6736(18)32552-2

4. Lee EK, Matulonis UA. PARP Inhibitor Resistance Mechanisms and Implications for Post-Progression Combination Therapies. Cancers (Basel). 2020;12(8):2054. doi:10.3390/cancers12082054

5. Elattar A, Bryant A, Winter-Roach BA, Hatem M, Naik R. Optimal primary surgical treatment for advanced epithelial ovarian cancer. Cochrane Database Syst Rev. 2011;2011(8):CD007565. doi:10.1002/14651858.CD007565. pub2

6. Colombo N, Sessa C, du Bois A, et al. ESMO-ESGO consensus conference recommendations on ovarian cancer: pathology and molecular biology, early and advanced stages, borderline tumours and recurrent disease ${ }^{\dagger}$. Ann Oncol. ne tumours and recurrent disease. Ann

7. Matulonis UA, Sood AK, Fallowfield L, Howitt BE, Sehouli J, Karlan BY. Ovarian cancer. Nat Rev Dis Primer. 2016;2:16061. doi: $10.1038 /$ nrdp.2016.61

8. Tew WP, Lacchetti C, Ellis A, et al. PARP Inhibitors in the Management of Ovarian Cancer: ASCO Guideline. J Clin Oncol.2020;38(30):3468-3493. doi:10.1200/JCO.20.01924

9. Moore K, Colombo N, Scambia G, et al. Maintenance Olaparib in Patients with Newly Diagnosed Advanced Ovarian Cancer. N EnglJ Med. 2018;379(26):2495-2505. doi:10.1056/ NEJMoa1 1810858

10. González-Martín A, Pothuri B, Vergote I, et al. Niraparib in Patients with Newly Diagnosed Advanced Ovarian Cancer. $N$ Engl J Med. 2019;381(25):2391-2402. doi:10.1056/NEJMoal 1910962

11. Ray-Coquard I, Pautier P, Pignata S, et al. Olaparib plus Bevacizumab as First-Line Maintenance in Ovarian Cancer. $N$ Engl J Med. 2019;381(25):2416-2428. doi:10.1056/NEJMoa1911361

12. Poveda A, Floquet A, Ledermann JA, et al. Olaparib tablets 12. Poveda A, Floquet A, Ledermann JA, et al. Olaparib tablets as maintenance therapy in patients with platinum-sensitive relapsed ovarian cancer and a BRCA1/2 mutation (SOLO2/
ENGOT-Ov21): a final analysis of a double-blind, randomised, placebo-controlled, phase 3 trial. Lancet Oncol. 2021;22(5):620631. doi:10.1016/S1470-2045(21)00073-5

13. Mirza MR, Monk BJ, Herrstedt J, et al. Niraparib Maintenance Therapy in Platinum-Sensitive, Recurrent Ovarian Cancer. $N$ Engl J Med. 2016:375(22):2154-2164. doi:10.1056/ NEJMoal 611310

14. Coleman RL, Oza AM, Lorusso D, et al. Rucaparib mainte14. Coleman RL, Oza AM, Lorusso D, et al. Rucaparib mainteto platinum therapy (ARIEL3): a randomised, double-blind, placebo-controlled, phase 3 trial. Lancet. 2017;390(10106):19491961. doi:10.1016/S0140-6736(17)32440-6

15. Moore KN, Secord AA, Geller MA, et al. Niraparib monotherapy for late-line treatment of ovarian cancer (QUADRA): a multicentre, open-label, single-arm, phase 2 trial. Lancet Oncol. 2019;20(5):636-648. doi:10.1016/S1470-2045(19)30029-4 16. Penson RT, Valencia RV, Cibula D, et al. Olaparib Versus Nonplatinum Chemotherapy in Patients With Platinum-Sensitive Relapsed Ovarian Cancer and Germline BRCA1/2 Mutation (SOLO3): A Randomized Phase III Trial. J Clin Oncol. tion (SOLO3): A Randomized Phase III Trial. J Clin O 2020;38(11):1164-1174. doi:10.1200/JCO.19.02745 17. McMullen M, Karakasis K, Madariaga A, Oza AM. Over-
coming Platinum and PARP-Inhibitor Resistance in Ovarian Cancer. Cancers (Basel). 2020;12(6):1607. doi:10.3390/cancers 12061607

18. Chiappa M, Guffanti F, Bertoni F, Colombo I, Damia G. Overcoming PARPi resistance: Preclinical and clinical evidence in ovarian cancer. Drug Resist Updat. 2021;55:100744. doi:10.1016/j.drup.2021.100744
19. Goel N, Foxall ME, Scalise CB, Wall JA, Arend RC. Strategies in Overcoming Homologous Recombination Proficiency and PARP Inhibitor Resistance. Mol Cancer The 2021;20(9):1542-1549. doi:10.1158/1535-7163.MCT-200992

20. Lord CJ, Ashworth A. PARP inhibitors: Synthetic lethality in the clinic. Science. 2017;355(6330):1152-1158. doi:10.1126 science.aam 7344

21. Ashworth A. A synthetic lethal therapeutic approach: poly(ADP) ribose polymerase inhibitors for the treatment of cancers deficient in DNA double-strand break repair.J Clin Oncol. cers deficient in DNA double-strand break repair.J Clin Onco

22. Konstantinopoulos PA, Ceccaldi R, Shapiro GI, D’Andrea AD. Homologous Recombination Deficiency: Exploiting the Fundamental Vulnerability of Ovarian Cancer. Cancer Disco 2015;5(11):1137-1154. doi:10.1158/2159-8290.CD-15-0714 23. Miller RE, Leary A, Scott CL, et al. ESMO recommendations on predictive biomarker testing for homologous recombination deficiency and PARP inhibitor benefit in ovarian cance. Ann Oncol. 2020;31(12):1606-1622. doi:10.1016/j.annonc. 2020.08 .2102

24. Tobalina L, Armenia J, Irving E, O'Connor MJ, Forment JV. A meta-analysis of reversion mutations in BRCA genes identifies signatures of DNA end-joining repair mechanisms driving therapy resistance. Ann Oncol. 2021;32(1):103-112. doi:10.1016/j.annonc.2020.10.470

25. Norquist B, Wurz KA, Pennil CC, et al. Secondary somatic mutations restoring $\mathrm{BRCA} 1 / 2$ predict chemotherapy resistance in hereditary ovarian carcinomas. I Clin Oncol. 2011;29(22):3008-3015. doi:10.1200/JCO.2010.34.2980 26. Lheureux S, Bruce JP, Burnier JV, et al. Somatic BRCA1/2 Recove Polymerase Inhibition. J Clin On. Pol 10.1200/JCO.201671.3677 27. Bouwman P, Aly A, Escandell JM, et al. 53BP1 loss rescues BRCA1 deficiency and is associated with triple-negative an BRCA-mutated breast cancers. Nat Struct Mol Biol. 2010;17(6):688-695. doi:10.1038/nsmb.1831

28. Cardnell RJ, Feng Y, Diao L, et al. Proteomic markers of DNA repair and PI3K pathway activation predict response to the PARP inhibitor BMN 673 in small cell lung cancer. Clin Cancer Res. 2013;19(22):6322-6328. doi:10.1158/1078-0432. CCR-13-1975

29. Sun C, Fang Y, Yin J, et al. Rational combination therapy with PARP and MEK inhibitors capitalizes on therapeutic liabilities in RAS mutant cancers. Sci Transl Med. 2017;9(392):eaal5148. doi:10.1126/scitranslmed.aal5148 30. Pilié PG, Tang C, Mills GB, Yap TA. State-of-the-art strategies for targeting the DNA damage response in cancer. Nat Rev Clin Oncol. 2019;16(2):81-104. doi:10.1038/s41571-0180114-z

31. Ghelli Luserna di Rorà $\mathrm{A}$, Cerchione $\mathrm{C}$, Martinelli G, Simonetti G. A WEE 1 family business: regulation of mitosis, can cer progression, and therapeutic target. I Hematol Oncol. cer progression, and therapeutic target. J Hem

32. Iorns E, Lord CJ, Grigoriadis A, et al. Integrated functional, gene expression and genomic analysis for the identification of cancer targets. PLoS One. 2009;4(4):e5120. doi:10.1371/journal.pone. 0005120

33. Slipicevic A, Holth A, Hellesylt E, Tropé CG, Davidson B, Flørenes VA. Weel is a novel independent prognostic marker of poor survival in post-chemotherapy ovarian carcinoma effusions. Gynecol Oncol. 2014;135(1):118-124. doi:10.1016/j.ygyno.2014.07.102

34. Backert S, Gelos M, Kobalz U, et al. Differential gene expression in colon carcinoma cells and tissues detected with cDNA array. Int J Cancer. 1999;82(6):868-874. doi:10.1002/ (sici) 1097-0215(19990909)82:6<868::aid-ijc16>3.0.co;2-w 35. Do K, Doroshow JH, Kummar S. Weel kinase as a target for cancer therapy. Cell Cycle. 2013;12(19):3159-3164. doi: $10.4161 /$ cc. 26062

36. Guertin AD, Li J, Liu Y, et al. Preclinical evaluation of the WEE1 inhibitor MK-1775 as single-agent anticancer therapy. Mol Cancer Ther. 2013;12(8):1442-1452. doi:10.1158/15357163.MCT-13-002
37. Gorecki L, Andrs M, Korabecny J. Clinical Candidates Tarenting ATR-CHK1-WEE1 Axis in Cancer. Cancers (Basel). 2021:13(4):795 doi:10.3390/cancers 13040795

38. Kausar T, Schreiber JS, Karnak D, et al. Sensitization of Pancreatic Cancers to Gemcitabine Chemoradiation by WEE Kinase Inhibition Depends on Homologous Recombination Repair. Neoplasia. 2015;17(10):757-766. doi:10.1016/j. neo.2015.09.006

39. Leijen S, van Geel RMJM, Pavlick AC, et al. Phase I Study Evaluating WEE1 Inhibitor AZD1775 As Monotherapy and in Combination With Gemcitabine, Cisplatin, or Carboplatin in Patients With Advanced Solid Tumors. I Clin Oncol. 2016:34(36):4371-4380. doi:10.1200/JCO.2016.67.5991 40. Do K, Wilsker D, Ji J, et al. Phase I Study of Single-Agen AZD1775 (MK-1775), a Weel Kinase Inhibitor, in Patient With Refractory Solid Tumors.J Clin Oncol. 2015;33(30):34093415. doi: 10.1200/JCO.2014.60.4009

41. Hirai H, Arai T, Okada M, et al. MK-1775, a small molecule Weel inhibitor, enhances anti-tumor efficacy of various DNA-damaging agents, including 5-Aluorouracil. Cancer Biol Ther. 2010;9(7):514-522. doi:10.4161/cbt.9.7.11115

42. Leijen S, van Geel RMJM, Sonke GS, et al. Phase II Study of WEE1 Inhibitor AZD1775 Plus Carboplatin in Patients With TP53-Mutated Ovarian Cancer Refractory or Resistant to First-Line Therapy Within 3 Months. J Clin Oncol. 2016;34(36):4354-4361. doi:10.1200/JCO.2016.67.5942 43. Lheureux S, Cristea MC, Bruce JP, et al. Adavosertib plus gemcitabine for platinum-resistant or platinum-refractory recurrent ovarian cancer: a double-blind, randomised, placebodoi:1010d, phase 2 trial. Lancet. 2021

44. Westin SN, Coleman RL, Fellman BM, et al. EFFORT: EFFicacy Of adavosertib in parp ResisTance: A randomized two-arm non-comparative phase II study of adavosertib with or without olaparib in women with PARP-resistant ovarian cancer $J$ Clin Oncol. 2021:39(15_suppl):5505. doi:10.1200/ JCO.2021.39.15_suppl.5505

45. Oza AM, Estevez-Diz M, Grischke EM, et al. A Biomarkerenriched, Randomized Phase II Trial of Adavosertib (AZD1775) Plus Paclitaxel and Carboplatin for Women with Platinum-sensitive TP53-mutant Ovarian Cancer. Clin Cancer Res. 2020;26(18):4767-4776. doi:10.1158/1078-0432.CCR20-0219

46. Karnak D, Engelke CG, Parsels LA, et al. Combined inhib tion of Weel and PARP1/2 for radiosensitization in pancreatic cancer. Clin Cancer Res. 2014;20(19):5085-5096. doi:10.1158/1078-0432.CCR-14-1038

47. Fang Y, McGrail DJ, Sun C, et al. Sequential Therapy with PARP and WEE 1 Inhibitors Minimizes Toxicity while Maintaning Efficacy. Cancer Cell. 2019;35(6):851-867.e7. doi:10.1016/j.ccell.2019.05.001

48. Cancer Genome Atlas Research Network, Kandoth C, Schultz N, et al. Integrated genomic characterization of endometrial carcinoma. Nature. 2013;497(7447):67-73. doi:10.1038/nature 12113

49. Liu JF, Xiong N, Campos SM, et al. Phase II Study of the WEE1 Inhibitor Adavosertib in Recurrent Uterine Serous Carcinoma. J Clin Oncol. 2021;39(14):1531-1539. doi:10.1200/ JCO. 20.03167

50. Liu JF, Herold C, Gray KP, et al. Assessment of Combined Nivolumab and Bevacizumab in Relapsed Ovarian Cancer: A Phase 2 Clinical Trial. JAMA Oncol. 2019;5(12):1731-1738. doi:10.1001/jamaoncol.2019.3343 